\title{
Perfil cognitivo e de saúde de idosos de um Centro de Convivência
}

\author{
Cognitive and health profile of elderly of a Coexistence Center \\ Perfil cognitivo y de salud de ancianos de un Centro de Convivencia
}

Recebido: 12/09/2021 | Revisado: 19/09/2021 | Aceito: 23/09/2021 | Publicado: 25/09/2021

Mayse Cristelle de Sales Mélo ORCID: https://orcid.org/0000-0002-9402-0752 Universidade Federal de Campina Grande, Brasil E-mail: mayse.csm14@hotmail.com

José Antonio da Silva Júnior ORCID: https://orcid.org/0000-0001-7965-3095 Universidade Federal de Campina Grande, Brasil E-mail: joseantonio.030@ hotmail.com

Josefa Raquel Luciano da Silva

ORCID: https://orcid.org/0000-0003-1791-5541 Universidade Federal de Campina Grande, Brasil E-mail: jraquel.silva@hotmail.com

Nayanne Leal do Monte

ORCID: https://orcid.org/0000-0002-2750-4095 Centro Universitário UNIFACISA, Brasil

E-mail: nayannelealm@gmail.com

Heloísa Souto Policarpo Araújo ORCID: https://orcid.org/0000-0001-6041-0595 Universidade Federal de Campina Grande, Brasil E-mail: heloisasouto15@gmail.com

Natally Calixto Lucena

ORCID: https://orcid.org/0000-0002-3619-1545 Universidade Federal de Campina Grande, Brasil E-mail: natally_calixto@hotmail.com

Brenda Sales Lins

ORCID: https://orcid.org/0000-0002-4552-1788 Universidade Federal de Campina Grande, Brasil E-mail: salesbrenda0@gmail.com

Fabíola de Araújo Leite Medeiros ORCID: https://orcid.org/0000-0002-0834-1155 Universidade Federal de Campina Grande, Brasil E-mail: profabiola@bol.com.br

Ana Elisa Pereira Chaves

ORCID: https://orcid.org/0000-0002-1960-0984 Universidade Federal de Campina Grande, Brasil E-mail: aepchaves@gmail.com

Ana Cláudia Torres de Medeiros ORCID: https://orcid.org/0000-0002-3695-9745 Universidade Federal de Campina Grande, Brasil E-mail: anaclaudia.tm@hotmail.com

\begin{abstract}
Resumo
Objetivo: Identificar o perfil cognitivo e as condições de saúde de idosos participantes de um Centro de Convivência do Idoso no município de Campina Grande/PB. Metodologia: Trata-se de um estudo descritivo e transversal, com abordagem quantitativa. Foi utilizado como instrumento de coleta de dados um questionário sociodemográfico e o Mini Exame do Estado Mental. Para a análise dos dados, foi utilizada a estatística descritiva. Resultados: Houve predomínio do sexo feminino $60,7 \%$, faixa etária entre 70 a 79 anos $35,7 \%$, viúvos $44,7 \%$, um a quatro anos de escolaridade 53,6\%, residem acompanhados 83,9\%. Com relação as morbidades autorreferidas, destacaram-se hipertensão arterial sistêmica e diabetes mellitus. Por meio do Mini Exame do Estado Mental constatou-se que 44,6\% dos idosos apresentam declínio cognitivo quando analisado o grupo total, sendo mais predominante em mulheres. Conclusões: Espera-se que esses resultados subsidiem profissionais e estudiosos da área da gerontologia, com relação a caracterização do perfil sociodemográfico e cognitivo de idosos participantes de Centros de Convivência, e que também auxilie no planejamento de atividades e acompanhamento profissional.
\end{abstract}


Palavras-chave: Cognição; Centros comunitários para idosos; Demência; Idoso.

\begin{abstract}
Objective: To identify the cognitive profile and health conditions of elderly participants in a Living Center for the Elderly in the city of Campina Grande/PB. Methodology: This is a descriptive and cross-sectional study with a quantitative approach. A sociodemographic questionnaire and the Mini Mental State Examination were used as a data collection instrument. For data analysis, descriptive statistics was used. Results: There was a predominance of females $60.7 \%$, aged between 70 and 79 years old $35.7 \%$, widowers $44.7 \%$, one to four years of schooling $53.6 \%$, $83.9 \%$ lived together. Regarding self-reported morbidities, systemic arterial hypertension and diabetes mellitus stood out. Through the Mini Mental State Examination it was found that $44.6 \%$ of the elderly presented cognitive decline when analyzing the total group, being more predominant in women. Conclusions: It is hoped that these results support professionals and scholars in the field of gerontology, with respect to the characterization of the sociodemographic and cognitive profile of elderly participants in Community Centers, and that it also helps in planning activities and professional monitoring.
\end{abstract}

Keywords: Cognition; Community centers for the elderly; Insanity; Old man.

\title{
Resumen
}

Objetivo: Identificar el perfil cognitivo y las condiciones de salud de los ancianos participantes en un Centro de Vida para Personas Mayores de la ciudad de Campina Grande/PB. Metodología: Se trata de un estudio descriptivo, transversal con enfoque cuantitativo. Se utilizó un cuestionario sociodemográfico y el Mini Examen del Estado Mental como instrumento de recolección de datos. Para el análisis de datos se utilizó estadística descriptiva. Resultados: Predominó el sexo femenino $60,7 \%$, edad entre 70 y 79 años $35,7 \%$, viudos $44,7 \%$, de uno a cuatro años de escolaridad 53,6\%, 83,9\% convivían. En cuanto a las morbilidades autoinformadas, se destacaron la hipertensión arterial sistémica y la diabetes mellitus. A través del Mini Examen del Estado Mental se encontró que el 44,6\% de los ancianos presentó deterioro cognitivo al analizar el grupo total, siendo más predominante en las mujeres. Conclusiones: Se espera que estos resultados apoyen a los profesionales y académicos del campo de la gerontología, con respecto a la caracterización del perfil sociodemográfico y cognitivo de los adultos mayores participantes en los Centros Comunitarios, y que también ayuden en la planificación de actividades y seguimiento profesional.

Palabras clave: Cognición; Centros comunitarios para personas mayores; Locura; Anciano.

\section{Introdução}

Mundialmente, observa-se um crescimento populacional de pessoas com idade igual ou maior a 60 anos, ocorrendo de forma mais rápida que as demais faixas etárias. Para tanto, considera-se como idoso, em países em desenvolvimento, aqueles indivíduos com faixa etária a partir dos 60 anos de idade e em países desenvolvidos, a partir de 65 anos de idade.

O número de pessoas com 60 anos ou mais deve duplicar entre 2007 e 2050, e mais que triplicar em 2050, alcançando dois bilhões. Ressalta-se que esse fato é resultante da diminuição do número de nascimentos e aumento da expectativa de vida. Esse significativo aumento da expectativa de vida relaciona-se com a diminuição das doenças transmissíveis contrapondo-se a elevação das doenças crônicas não-transmissíveis.

A partir dos 60 anos de idade, o indivíduo pode apresentar perdas de audição, visão e movimentos relacionados à idade, bem como doenças crônicas não transmissíveis, incluindo doenças cardíacas, acidente vascular encefálico, doenças respiratórias crônicas, câncer e demência, os quais levam a limitações funcionais e ao falecimento.

Com o envelhecimento biológico, o Sistema Nervoso Central é um dos mais afetados, assim, aumenta-se o risco de desenvolvimento de declínio cognitivo, que ocorre por meio do processo de degeneração do sistema nervoso incluindo diversas alterações estruturais como hipertrofia cerebral e alterações no sistema de neurotransmissores. Ademais, essas mudanças são influenciadas por fatores culturais, genéticos, presença de comorbidades e estilo de vida do idoso.

As alterações cognitivas, frequentemente estão relacionadas à atenção, memória, linguagem, velocidade de processamento executivo e psicomotor. No Brasil, estima-se que 5\% a 30\% dos idosos apresentam quadros demenciais, que interferem em sua funcionalidade e qualidade de vida. 
No mundo, mais de 35 milhões de pessoas tinham algum grau de demência em 2010, e estima-se que em 2050, o número ultrapasse de 115 milhões de pessoas com algum grau de demência. Aproximadamente 60\% dos indivíduos afetados por demência, são de países em desenvolvimento, resultando em um maior planejamento e investimento em atenção à saúde, já que essas doenças acarretam grande impacto econômico e social. Ressalta-se, que em todo o mundo cerca de 50 milhões de pessoas sofrem com demência e anualmente 10 milhões de novos casos são diagnosticados.

A demência é entendida como uma síndrome, que geralmente possui natureza crônica ou progressiva, caracterizada por comprometimento da função cognitivade forma anormal para o que é considerado uma consequência do envelhecimento fisiológico. A demência afeta a memória, pensamento, orientação, compreensão, cálculo, capacidade de aprendizagem, linguagem e julgamento.

Destaca-se que, mundialmente, o custo resultante do tratamento de pessoas com demência atualmente é de US\$ 818 bilhões, o equivalente a mais de $1 \%$ do produto interno bruto global custa mais de US\$ 604 bilhões por ano; estando incluso o custo da prestação de cuidados de saúde e sociais, bem como a redução ou perda de renda de pessoas com demência e seus cuidadores, até 2030, espera-se que esse valor chegue a US\$ 2 trilhões, montante que pode prejudicar o desenvolvimento social e econômico e sobrecarregar os serviços sociais e de saúde.

Desse modo, diante do processo de envelhecimento, destaca-se no campo da atenção à saúde o declínio da capacidade cognitiva em idosos, o qual gera uma atenção maior a esse grupo populacional. O Mini Exame do Estado Mental (MEEM) é considerado o teste de rastreio cognitivo mais utilizado no mundo em idosos e adultos com versões traduzidas e autorizadas para vários países do mundo. Esse instrumento avalia determinadas atividades cognitivas, classificando-as em sete categorias com pontuação que varia do zero a trinta pontos subdivididos em questões sobre orientação temporal e espacial, atenção, memória, linguagem e produção visual.

Diante desse cenário, torna-se também um desafio em termos de políticas públicas, atender o crescente número de pessoas com demência. Uma ampla abordagem no setor da saúde pública torna-se necessária para melhorar o atendimento e a qualidade de vida das pessoas com demência bem como seus cuidadores e familiares.

Ressalta-se que diante do crescimento do número de idosos acometidos com doenças crônicas e com declínio cognitivo, os Centros de Convivência de idosos ganham um importante papel social, já que promovem a melhoria das condições de saúde dos mesmos. Segundo a portaria $n^{\circ} 73$ da Secretaria de Estado de Assistência Social/Ministério da Previdência e Assistência Social, de 10 de maio de 2001, os Centros de Convivência proporcionam atendimento fortalecendo atividades que, contribuem para autonomia, envelhecimento ativo e saudável, prevenção do isolamento social, socialização e aumento da renda própria. Sendo o espaço destinado a frequência dos idosos e de seus familiares, onde são planejadas e desenvolvidas ações de atenção ao idoso.

Considerando os aspectos apresentados este estudo objetivou identificar o perfil cognitivo e as condições de saúde de idosos participantes de um Centro de Convivência.

\section{Metodologia}

Trata-se de um estudo descritivo e transversal, de abordagem quantitativa, realizado no Centro Municipal de Convivência do Idoso, localizado no município de Campina Grande, Paraíba, Brasil. Os estudos transversais têm por objetivo obter dados fidedignos a fim de, ao final do estudo, elaborar conclusões robustas e confiáveis, bem como poder gerar novas hipóteses que poderão ser avaliadas em novos estudos (Zangirolami-Raimundo, et al., 2018).

Em relação aos critérios de elegibilidade, foram incluídas pessoas com idade igual ou superior a 60 anos, de ambos os sexos que estivessem participando do Centro de Convivência há pelo menos seis meses. Foram excluídos os indivíduos que 
apresentaram déficits de audição ou de visão graves; diagnóstico de déficits cognitivos graves e/ou doenças mentais que pudessem impedir o entendimento e a execução do estudo.

O Centro de Convivência possui 65 idosos que participam regularmente das atividades de propostas. O tamanho da amostra foi definido considerando o cálculo para amostra finita com proporções conhecidas, obtendo-se uma margem de erro de $5 \%$ (Erro=0,05), grau de confiabilidade de 95\% $(\alpha=0,05$, que fornece $Z 0,05 / 2=1,96)$, totalizando uma amostra de 56 participantes selecionados aleatoriamente. A coleta de dados ocorreu no período de fevereiro a abril de 2019.

A coleta de dados foi subsidiada por um roteiro estruturado para a obtenção das informações sociodemográficas (sexo, idade, estado civil, escolaridade, arranjo domiciliar, situação de atividade, raça/cor, situação de moradia, religião, renda familiar, motivo por ter procurado participar do serviço) e relacionadas aos aspectos clínicos (presença de morbidades e uso de medicamentos), além da escala do MEEM, para avaliar o estado cognitivo.E por fim, para confirmação do tratamento medicamentoso relatado, foi consultado o prontuário médico de cada idoso participante do estudo.

O MEEM é composto por questões agrupadas nos domínios, cada uma delas com o objetivo de avaliar um grupo de funções cognitivas específicas: orientação temporal (5 pontos); orientação espacial (5 pontos); memória imediata (3 pontos); atenção e cálculo (5 pontos); memória de evocação (3 pontos); linguagem (8 pontos); e capacidade construtiva visual (1 ponto). A pontuação total pode variar de zero até 30 pontos. Foi realizada a classificação (idosos com ou sem declínio cognitivo), conforme os pontos de corte propostos pelos autores (Brucki et al., 2003) para analfabetos, 20; para idade de 1 a 4 anos, 25; de 5 a 8 anos, 26,5; de 9 a 11 anos, 28; para indivíduos com escolaridade superior a 11 anos, 29. Assim, foram considerados dois grupos para a análise dos dados, em função do prejuízo cognitivo.

Ainda, o Mini Exame do Estado Mental (MEEM) é a escala mais utilizada para o rastreamento do comprometimentocognitivo. Clinicamente, o MEEM tem sido usado para detecção e acompanhamento da evolução de alterações cognitivas, bem como para o monitoramento de eficácia e de efetividade de tratamentos dessas alterações (Valle, 2009).

Após a coleta, os dados foram digitados e organizados em um banco de dados do programa Microsoft Office Excel for Windows 2013, utilizando estatística descritiva simples. Posteriormente, os resultados foram discutidos de acordo com a literatura pertinente a temática.

O estudo respeitou os aspectos éticos preconizados pela Resolução no 466/12 do Conselho Nacional de Saúde, tendo sido avaliado pelo Comitê de Ética em Pesquisa do Hospital Universitário Alcides Carneiro, o qual recebeu parecer favorável sob o Certificado de Apresentação para Apreciação Ética de número 03908418.5.0000.5182 e sob parecer de número 3.080.374 Ministério da Saúde (2012).

\section{Resultados}

A Tabela 1 contém as características sociodemográficas da população do estudo a partir da raça/cor, gênero, faixa etária, estado civil, escolaridade, religião, renda familiar, ocupação e características de moradia. 
Tabela 1: Caracterização dos idosos participantes do Centro de Convivência segundo as variáveis sociodemográficas. Campina Grande-PB, 2019.

\begin{tabular}{|c|c|c|c|c|c|c|}
\hline \multirow{2}{*}{ Variáveis } & \multicolumn{2}{|c|}{$\mathrm{CDC}^{*}$} & \multicolumn{2}{|c|}{ SDC $^{* *}$} & \multicolumn{2}{|c|}{ Total } \\
\hline & $\mathbf{N}$ & $\%$ & $\mathbf{N}$ & $\%$ & $\mathbf{N}$ & $\%$ \\
\hline \multicolumn{7}{|l|}{ Raça/cor } \\
\hline Pardo(a) & 14 & 56,0 & 16 & 51,6 & 30 & 53,6 \\
\hline Branco(a) & 07 & 28,0 & 10 & 32,3 & 17 & 30,4 \\
\hline Preto(a) & 01 & 4,0 & 03 & 9,7 & 04 & 7,1 \\
\hline Amarelo(a) & 01 & 4,0 & 02 & 6,4 & 03 & 5,3 \\
\hline Indígena & 02 & 8,0 & 00 & 0,0 & 02 & 3,6 \\
\hline \multicolumn{7}{|l|}{ Gênero } \\
\hline Feminino & 17 & 68,0 & 17 & 54,8 & 34 & 60,7 \\
\hline Masculino & 08 & 32,0 & 14 & 45,2 & 22 & 39,3 \\
\hline \multicolumn{7}{|l|}{ Faixa etária } \\
\hline De 60 a 69 anos & 07 & 28,0 & 12 & 38,7 & 19 & 33,9 \\
\hline De 70 a 79 anos & 08 & 32,0 & 12 & 38,7 & 20 & 35,7 \\
\hline Maior ou igual a 80 anos & 10 & 40,0 & 07 & 22,6 & 17 & 30,4 \\
\hline \multicolumn{7}{|l|}{ Estado civil } \\
\hline Casado(a) & 05 & 20,0 & 14 & 45,2 & 19 & 33,9 \\
\hline Solteiro(a) & 06 & 24,0 & 02 & 6,4 & 08 & 14,3 \\
\hline Viúvo(a) & 13 & 52,0 & 12 & 38,7 & 25 & 44,7 \\
\hline Divorciado(a) & 01 & 4,0 & 03 & 9,7 & 04 & 7,1 \\
\hline \multicolumn{7}{|l|}{ Escolaridade } \\
\hline Analfabeto & 04 & 16,0 & 09 & 29,1 & 13 & 23,2 \\
\hline 1 a 4 anos & 13 & 52,0 & 17 & 54,9 & 30 & 53,6 \\
\hline 5 a 8 anos & 05 & 20,0 & 02 & 6,4 & 07 & 12,5 \\
\hline 9 a 11 anos & 01 & 4,0 & 01 & 3,2 & 02 & 3,6 \\
\hline Mais de 11 anos & 02 & 8,0 & 02 & 6,4 & 04 & 7,1 \\
\hline \multicolumn{7}{|l|}{ Religião } \\
\hline Católica & 24 & 96,0 & 25 & 80,7 & 49 & 87,5 \\
\hline Evangélica & 01 & 4,0 & 05 & 16,1 & 06 & 10,7 \\
\hline \multicolumn{7}{|l|}{ Renda familiar**** } \\
\hline Menos de 1 SM & 01 & 4,0 & 00 & 0,0 & 01 & 1,8 \\
\hline Um SM & 10 & 40,0 & 05 & 16,1 & 15 & 26,8 \\
\hline Três ou mais SM & 03 & 12,0 & 07 & 22,6 & 10 & 17,8 \\
\hline \multicolumn{7}{|l|}{ Ocupação } \\
\hline Aposentado(a) & 24 & 96,0 & 29 & 93,6 & 53 & 94,8 \\
\hline Do lar & 01 & 4,0 & 01 & 3,2 & 02 & 3,6 \\
\hline Pensionista & 00 & 0,0 & 01 & 3,2 & 01 & 1,8 \\
\hline \multicolumn{7}{|l|}{ Mora sozinho } \\
\hline Não & 20 & 80,0 & 27 & 87,1 & 47 & 83,9 \\
\hline \multicolumn{7}{|l|}{ Responsabilidade sobre a moradia } \\
\hline Do idoso & 17 & 68,0 & 23 & 74,2 & 40 & 71,4 \\
\hline Dos filhos & 06 & 24,0 & 03 & 9,7 & 09 & 16,1 \\
\hline
\end{tabular}




\begin{tabular}{|c|c|c|c|c|c|c|}
\hline Gênero & 01 & 4,0 & 01 & 3,2 & 02 & 3,6 \\
\hline Irmão(a) & 01 & 4,0 & 01 & 3,2 & 02 & 3,6 \\
\hline Cônjuge & 00 & 0,0 & 03 & 9,7 & 03 & 5,3 \\
\hline \multicolumn{7}{|c|}{ Condição de moradia } \\
\hline Aluguel & 04 & 16,0 & 04 & 12,9 & 08 & 14,3 \\
\hline Casa própria & 21 & 84,0 & 26 & 83,9 & 47 & 83,9 \\
\hline Cedida & 00 & 0,0 & 01 & 3,2 & 01 & 1,8 \\
\hline
\end{tabular}

* CDC: Com declínio cognitivo segundo o MEEM

**SDC: sem declínio cognitivo segundo o MEEM

***SM: Salário mínimo nacional vigente na época da coleta de dados (2019): R \$ 998,00.

Fonte: Dados da pesquisa de autoria própria (2019).

A Tabela 1 evidencia o predomínio de mulheres, onde 34 (60,7\%)eram do gênero feminino e 22 (39,3\%) do gênero masculino; 20 (35,7\%) com faixa etária de 70 a 79 anos; 30 (53,6\%) se autodeclararam de raça/cor parda; 25 (44,7\%) viúvos, $30(53,6 \%)$ com escolaridade de 1 a 4 anos; 49 (87,5\%) de religião católica; 30 (53,6\%) com renda familiar de dois SM; 53 $(94,8 \%)$ tendo como principal fonte de renda aposentadoria; $47(83,9 \%)$ residem acompanhado; $47(83,9 \%)$ possuindo moradia própria e $40(71,4 \%)$ relataram ser responsável pela casa.

Nesta amostra, obteve-se média do MEEM de 23 pontos $(\mathrm{DP}=3,97)$, sendo que a pontuação pode variar de zero a 30 pontos. Houve o predomínio do índice sugestivo de declínio da capacidade cognitiva em 25 participantes (44,6\%), sendo 13 $(52,0 \%)$ entre os idosos com escolaridade entre um a quatro anos; 17 (68,0\%) do gênero feminino; cor/raça autodeclarada parda $14(56,0 \%) ; 10(40,0 \%)$ no subgrupo etário (maior ou igual a 80 anos) e;quanto ao estado civil, o maior percentual de declínio cognitivo esteve presente, nos idosos viúvos 13 (52,0\%) (Tabela 1).

A Tabela 2 descreve característica importantes acerca das condições de saúde dos idosos participantes como aos motivos para frequentar o centro de convivência, morbidades autorreferidas e medicações em uso.

Tabela 2: Características das condições de saúde dos idosos participantes do Centro de Convivência. Campina Grande-PB, 2019.

\begin{tabular}{|c|c|c|c|c|c|c|}
\hline \multirow{2}{*}{ Variáveis } & \multicolumn{2}{|c|}{$\mathrm{CDC}^{*}$} & \multicolumn{2}{|c|}{ SDC $^{* *}$} & \multicolumn{2}{|c|}{ Total } \\
\hline & $\mathbf{N}$ & $\%$ & $\mathbf{N}$ & $\%$ & $\mathbf{N}$ & $\%$ \\
\hline \multicolumn{7}{|c|}{ Motivo de frequentar o Centro de Convivência } \\
\hline Problemas de saúde & 02 & 8,0 & 01 & 3,2 & 03 & 5,3 \\
\hline Participar das atividades & 21 & 84,0 & 23 & 74,2 & 44 & 78,6 \\
\hline Orientação profissional & 00 & 0,0 & 01 & 3,2 & 01 & 1,8 \\
\hline Sair de casa & 01 & 4,0 & 02 & 6,4 & 03 & 5,3 \\
\hline Outros & 01 & 4,0 & 04 & 12,9 & 05 & 9,0 \\
\hline Diabetes mellitus & 10 & 40,0 & 12 & 38,7 & 22 & 39,3 \\
\hline Cardiopatia & 02 & 8,0 & 05 & 16,1 & 07 & 12,5 \\
\hline Insônia & 08 & 32,0 & 10 & 32,3 & 18 & 32,1 \\
\hline Catarata & 03 & 12,0 & 04 & 12,9 & 07 & 12,5 \\
\hline Problemas na coluna & 03 & 12,0 & 05 & 16,1 & 08 & 14,3 \\
\hline Dificuldade visual & 09 & 36,0 & 12 & 38,7 & 21 & 37,5 \\
\hline Ansiedade & 08 & 32,0 & 12 & 38,7 & 20 & 35,7 \\
\hline Depressão & 02 & 8,0 & 02 & 6,4 & 04 & 7,1 \\
\hline
\end{tabular}




\section{Problemas articulares}

Incontinência urinária

Neoplasia de mama

Osteoporose

Doença vascular periférica

Dificuldade auditiva

Constipação

Neoplasia de próstata

Hiperplasia prostática benigna

Nenhuma

$\begin{array}{llllll}04 & 16,0 & 04 & 12,9 & 08 & 14,3 \\ 01 & 4,0 & 01 & 3,2 & 02 & 2,6 \\ 01 & 4,0 & 01 & 3,2 & 02 & 2,6 \\ 00 & 0,0 & 02 & 6,4 & 02 & 2,6 \\ 00 & 0,0 & 02 & 6,4 & 02 & 2,6 \\ 00 & 0,0 & 02 & 6,4 & 02 & 2,6 \\ 01 & 4,0 & 02 & 6,4 & 03 & 5,3 \\ 01 & 4,0 & 01 & 3,2 & 02 & 2,6 \\ 01 & 4,0 & 01 & 3,2 & 02 & 2,6 \\ 01 & 4,0 & 00 & 0,0 & 01 & 1,8\end{array}$

\section{Classes de medicamentos utilizados}

Hipoglicemiantes orais

Hipolipêmicos

Anti-hipertensivos

Diuréticos

Insulina

Anti-inflamatórios não esteroidais

Antirreumáticos

Benzodiazepínicos

Antidepressivos

Controladores hormonais

Bifosfanatos

Antiácidos

Flebotômicos

Antiarrítmicos

Neurolépticos

Controlador do humor

Vasodilatadores

Antiagregantes plaquetários

10

11

10

40,0

11

$35,5 \quad 21$

37,5

$$
\text { 44,0 }
$$

10

$32,3 \quad 21$

37,5

$68,0 \quad 20$

$64,5 \quad 37$

$40,0 \quad 14$

$45,2 \quad 24$

12,0

02

$6,4 \quad 05$

01

* CDC: Com declínio cognitivo segundo o MEEM

**SDC: sem declínio cognitivo segundo o MEEM

Fonte: Dados da pesquisa de autoria própria (2019).

A Tabela 2 mostra que dentre os principais motivos que levaram os idosos a frequentar o Centro de Convivência, 44 $(78,6 \%)$ declararam estar associado a participação em atividades. No tocante às morbidades autorreferidas, observou-se predomínio da hipertensão arterial sistêmica (HAS) em 44 (78,6\%), diabetes mellitus em 22 (39,3\%) e dificuldade visual em $21(37,5 \%)$. As classes de medicamentos mais utilizadas foram os anti-hipertensivos com 37 (66,1\%), seguido de diuréticos com 24 (42,8\%) e em seguida os hipolipêmicos e hipoglicemiantes orais com 21 (37,5\%).

Relacionado especificamente aos idosos que apresentaram algum declínio cognitivo, a hipertensão e o diabetes também prevaleceram nesse grupo isolado, sendo, respectivamente, 24 (96,0\%) e $10(40,0 \%)$ participantes portadores dessas doenças. Ainda na perspectiva dos idosos com declínio cognitivo, quanto à classe de medicamentos mais utilizadas, 
prevaleceram os anti-hipertensivos utilizados por $17(68,0 \%)$ idosos, hipolipêmicos por 11 (44,0\%) idosos, diuréticos e hipoglicemiantes orais por $10(40,0 \%)$ cada.

\section{Discussão}

Em relação ao gênero dos participantes da pesquisa, houve predomínio do gênero feminino. Isso foi encontrado em outros estudos e pode ser explicado por questões socioculturais, já que as mulheres apresentam maior cuidado com a saúde e qualidade de vida, além de serem mais participativas em ações coletivas e de pesquisa (Zortea, 2015; Annes et al., 2017; Freire et al., 2015; Felippe e Campos, 2015). Como também, foi trazido em outra pesquisa, há um maior número de participantes em grupos de convivência de idosos de pessoas do sexo feminino, e esse predomínio ocorre em todo o Brasil (Wichmann, 2013).

Com relação ao perfil cognitivo, verificou-se por meio do MEEM que 25 (44,6\%) dos participantes apresentaram pontuações abaixo das notas de corte propostas para a escolaridade descritas (Brucki et al., 2003). Servindo de alerta para a importância do cuidado com a capacidade cognitiva dos idosos, já que a capacidade cognitiva apresenta relação com a qualidade de vida. O que torna a assistência a saúde uma ferramenta importante na detecção precoce de sinais e sintomas relacionados à perda cognitiva (Ferreira et al., 2014).

Sendo detectado um número maior de declínio cognitivo em mulheres $(68,0 \%)$ do que em homens $(32,0 \%)$. Um fator que colabora para a um maior número de mulheres com declínio cognitivo em relação aos homens é a maior expectativa de vida, que para as mulheres em 2012 era de 78,3 anos e para os homens era de 71 anos (Instituto Brasileiro de Geografia e Estatística, 2012). Com o aumento da idade é mais evidente a prevalência do surgimento de declínio cognitivo (Nascimento et al., 2015). Sendo, o sexo feminino mais afetado com demência do que o sexo masculino (Organização Pan-Americana da Saúde, 2017). Ademais, estudos afirmam, que osmulheres tem maior predisposição para desenvolver doença de Alzheimer, pelo fato, de possuírem uma maior expectativa de vida (Oliveira et al., 2012).

No que diz respeito à faixa etária dos idosos, houve predomínio da faixa de 70-79 anos (35,7\%), fato esse que ocorreu em outro estudo, em que a maioria dos idosos entrevistados faziam parte dessa mesma faixa etária (Annes, 2012). Com relação ao declínio cognitivo, $10(40 \%)$ dos idosos com idade igual ou superior a 80 anos, apresentaram pontuações abaixo do proposto (Brucki, 2003). Como enfatizado anteriormente, percebe-se que o avançar da idade aumenta a prevalência de declínio cognitivo, como mostra um estudo, que evidenciou a presença de declínio cognitivo, sendo duas vezes maior naqueles com 80 anos do que com 60 anos (Holz, 2013). Em outra pesquisa também houve predomínio do declínio em idosos com idade maior ou igual à 80 anos (Nascimento, 2015).

Neste estudo, houve o predomínio de declínio cognitivo entre os idosos com escolaridade entre um e quatro anos, totalizando $13(52,0 \%)$ idosos. Pessoas idosas com até quatro anos de estudo apresentaram predomínio de 2,24 vezes maior de déficit do que aquelas com cinco ou mais anos de estudo (Zortea, 2015). Sabe-se que o aumento da escolaridade é um fator de proteção em relação as perdas cognitivas (Nascimento, 2015). A escolarização influencia na melhoria do processamento do raciocínio, atenção, inteligência, funções executivas e memória, assim, melhores resultados em testes como o MEEM advém de indivíduos com maiores níveis de escolaridade (Domiciano, 2014).Observa-se também, que o grau de escolaridade influência nas condições do envelhecimento, dessa forma, o nível de instrução das pessoas idosas influi diretamente nas condições de vida, de convivência, na idade da aposentadoria, nas condições econômicas e, evidentemente, nos estados de ânimo para enfrentar os desafios da vida diária(Instituto de Mayores y Servicios Sociales, 2011).

Destaca-se, que $13(52,0 \%)$ idosos participantes do estudo que apresentaram declínio cognitivo são viúvos. Um estudo realizado no estado do Rio Grande do Sul, Brasil, comparou o desempenho cognitivo de idosos com e sem 
companheiro(a), sendo que os primeiros possuíam prevalência $45 \%$ menor de declínio cognitivo, o que pode explicar os resultados do estudo (Zortea, 2015).

Identificou-se que49 (87,5\%) participantes, um número considerável de idosos, seguem a religião católica foram. Isso também foi encontrado em um estudo, em que $81,9 \%$ dos idosos entrevistados relataram ter como religião o catolicismo (Freire, 2015).

Observa-se a predominância da aposentadoria como principal fonte de renda, assimassemelhou-se com os resultados dos estudos, nos quais a aposentadoria predominou como renda principal entre os idosos. No que diz respeito à condição domiciliar e de morar sozinho ou acompanhado, o estudo mostrou que 47 (83,9\%) dos entrevistados residiam com alguém e possuía moradia própria, assim como em outros estudos realizados (Annes, 2017; Freire, 2015).

Identificou-se que $30(53,6 \%)$ do total dos entrevistados se autodeclararam da raça/cor parda. Durante o estudo, 14 $(56 \%)$ dos idosos que apresentaram pontuação inferior no MEEM, autodeclaram-se da raça/cor parda, o que também foi identificado em outro estudo em que o maior número de idosos com achados sugestivos de sintomas demenciais foram em idosos que relataram raça/cor não branca (Lini et al., 2016).

Sobre as morbidades autorreferidas observa-se no grupo total o predomínio da hipertensão arterial sistêmica e diabetes mellitus. Semelhante aos resultados encontrados em outras pesquisas, em que houve maior incidência de hipertensão arterial e diabetes mellitus (Zortea, 2015; Freire, 2015).

Com relação as morbidades que predominaram entre os idosos com diminuição da capacidade cognitiva, foram a hipertensão e diabetes. Estudos mostram a diabetes mellitus como fator de risco para o desenvolvimento do declínio cognitivo (Vargas et al., 2014; Lopes et al., 2018). Ademais, uma pesquisa mostra a hipertensão arterial como um importante fator de risco para o comprometimento da função cognitiva (Chaves, 2015). O que pode explicar a presença de diabetes mellitus em 10 $(40,0 \%)$ dos participantes com declínio cognitivo e da hipertensão arterial em 24 (96,0\%) dos idosos dessa pesquisa, que apresentaram notas de corte inferiores.

O uso de medicamentos utilizados pelos idosos, desse estudo, justifica-se principalmente, pelo predomínio das doenças crônicas nessa faixa etária, já que o uso de medicamentos é uma das principais formas de intervenção (Santos, 2013).

\section{Conclusões}

O envelhecimento populacional tem ocorrido de forma progressiva e rápida, por isso, é cada vez mais importante discutir sobre o assunto. Torna-se visível a possibilidade de envelhecer com qualidade de vida, porém, para isso é necessário que haja mobilização de toda a sociedade, para um maior desenvolvimento e efetivação de políticas públicas que englobem um envelhecimento ativo e saudável, que envolva ações de prevenção e promoção a saúde.

A presente pesquisa possibilitou alcançar o objetivo proposto de identificar o perfil cognitivo e as condições de saúde de idosos que participam de um Centro de Conivência do idosos no município de Campina Grande, Paraíba, Brasil. Ressalta-se a importância dos Centros de Convivência para idosos, que contribuem significativamente para o envelhecimento saudável, para a prevenção do isolamento e exclusão social destes indivíduos.Como também, é uma alternativa de apoio ao cuidado e atua de forma a reduzir a sobrecarga dos familiares de idosos.

Verificou-se por meio do MEEM que o percentual de idosos com declínio cognitivo foi de 25 (44,6\%) quando analisado o grupo total, sendo mais predominante em mulheres 17 (68\%) do que nos homens 08 (32\%). Dessa forma, percebese que o público feminino necessita de um maior acompanhamento e intervenções no que diz respeito à cognição. Ressalta-se um número considerável de idosos com índices sugestivos de declínio cognitivo, sugere-se uma melhor investigação desses idosos com relação a função cognitiva dos mesmos. 
As morbidades apresentadas pelos idosos foram predominantemente doenças crônicas, tais como a hipertensão arterial sistêmica e diabetes mellitus, o que foi justificado o uso de terapia medicamentosa (hipoglicemiantes orais, hipolipêmicos, diuréticos e anti-hipertensivos) entre os idosos. Salienta-se que as condições de saúde dos indivíduos são determinadas socialmente e, portanto, não podem ser desvinculadas dos modos, condições e estilos de vida destes, os quais, muitas vezes, são garantidos por meio de políticas públicas.

Acredita-se que esses resultados poderão subsidiar profissionais e estudiosos da área da gerontologia, com relação a caracterização do perfil sociodemográfico e cognitivo de idosos participantes de Centros de Convivência. Poderão também, auxiliar em um melhor planejamento de atividades e acompanhamento profissional aos idosos que apresentam declínio cognitivo e na prevenção dos demais idosos.

Todavia, sobre as limitações desse estudo, aponta-se seu caráter transversal, o que dificulta uma avaliação longitudinal das variantes investigadas; no fato de explorar uma realidade específica envolvendo apenas idosos que frequentam um Centro de Convivência, há poucos estudos recentes sobre a temática. Também, salienta-se que a identificação do perfil cognitivo foi realizada por um único instrumento que foi o MEEM, mas isto não invalida a importância dos resultados encontrados

Dessa forma, sugere-se e considera-se importante a realização de novos estudos científicos para aprofundamento da temática abordada nessa pesquisa, permitindo uma melhor compreensão sobre o uso do MEEM em idosos que participam de Centros de Convivência.

\section{Conflito de interesse}

Os autores declaram que não haver conflito de interesse.

\section{Referências}

Andrade, F. L. J. P. D., Lima, J. M. R. D., Fidelis, K. D. N. M., Jerez-Roig, J., \& Lima, K. C. D. (2017). Cognitive impairment and associated factors among institutionalized elderly persons in Natal, Rio Grande do Norte, Brazil. Revista Brasileira de Geriatria e Gerontologia, 20, 186-196.

Annes, L. M. B., Mendonça, H. G. S., Lima, F. M. D., Lima, M. D. A. S., \& Aquino, J. M. D. (2017). Perfil sociodemográfico e de saúde de idosas que participam de grupos de terceira idade em Recife, Pernambuco. Revista Cuidarte, 8(1), 1499-1508.

Brucki, S., Nitrini, R., Caramelli, P., Bertolucci, P. H., \& Okamoto, I. H. (2003). Sugestões para o uso do mini-exame do estado mental no Brasil. Arquivos de Neuro-psiquiatria, 61(3B), 777-781.

Chaves, A. S., Santos, A. M. D., Alves, M. T. S. S. D. B., \& Salgado, N. (2015). Associação entre declínio cognitivo e qualidade de vida de idosos hipertensos. Revista Brasileira de Geriatria e Gerontologia, 18, 545-556.

Domiciano, B. R., Braga, D. K. A. P., da Silva, P. N., de Vasconcelos, T. B., \& Macena, R. H. M. (2014). Escolaridade, idade e perdas cognitivas de idosas residentes em instituições de longa permanência. Revista Neurociências, 22(3), 330-336.

Felippe, L. A., \& Campos, D. M. (2016). Perfil da Fragilidade em Idosos Participantes de um Centro de Convivência em Campo Grande-MS. Journalof Health Sciences, 18(4), 224-8.

Ferreira, L. S., Pinho, M. D. S. P., Pereira, M. W. D. M., \& Ferreira, A. P. (2014). Perfil cognitivo de idosos residentes em Instituições de Longa Permanência de Brasília-DF. Revista Brasileira de Enfermagem, 67, 247-251.

Freire, G. V., da Silva, I. P., de Moura, W. B., Rocha, F. C. V., de Araújo Madeira, M. Z., \& Amorim, F. C. M. (2015). Perfil de idosos que frequentam um centro de convivência da terceira idade. Revista Interdisciplinar, 8(2), 11-19.

Gutierrez, B. A. O., Silva, H. S. D., Guimarães, C., \& Campino, A. C. (2014). Impacto econômico da doença de Alzheimer no Brasil: é possível melhorar a assistência e reduzir custos?. Ciência \& Saúde Coletiva, 19, 4479-4486.

Holz, A. W., Nunes, B. P., Thumé, E., Lange, C., \&Facchini, L. A. (2013). Prevalência de déficit cognitivo e fatores associados entre idosos de Bagé, Rio Grande do Sul, Brasil. Revista Brasileira de Epidemiologia, 16, 880-888. 
Instituto de Mayores y ServiciosSociales. Envejecimientoactivo. (2011). Llanco. Madrid: IMSERSO. https://www.imserso.es/InterPresent1/groups/imserso/documents/binario/8088_8089libroblancoenv.pdf

Leite, M. T., Hildebrandt, L. M., Kirchner, R. M., Winck, M. T., Silva, L. A. A. D., \& Franco, G. P. (2012). Estado cognitivo e condições de saúde de idosos que participam de grupos de convivência. Revista gaúcha de Enfermagem, 33, 64-71.

Lini, E. V., Lima, A. P. D., Giacomazzi, R. B., Doring, M., \& Portella, M. R. (2016). Prevalência e fatores associados aos sintomas sugestivos de demência em idosos. Cien. Cogn, 21(2), 189-97.

Lopes, C. M., Junior, J. C. D. S. M., Pessoa, I. A., da Silva Wan-Meyl, F., \&Burbano, R. M. R. (2018). Diabetes mellitus e a doença de Alzheimer. Arquivos Catarinenses de Medicina, 47(1), 159-168.

Melo, D. M. D., \& Barbosa, A. J. G. (2015). O uso do Mini-Exame do Estado Mental em pesquisas com idosos no Brasil: uma revisão sistemática. Ciência \& saúde coletiva, 20, 3865-3876.

Ministério da Saúde. (2012). Conselho Nacional de Saúde. Resolução n. 466. Dispõe sobre diretrizes e normas regulamentadoras de pesquisas envolvendo seres humanos.

Retirado de http://bvsms.saude.gov.br/bvs/saudelegis/cns/2013/res0466_12_12_2012.html

Moraes, E. N. D. (2018). Atenção à saúde do idoso: aspectos conceituais.

Nascimento, R. A. S. A. D., Batista, R. T. S., Rocha, S. V., \& Vasconcelos, L. R. C. (2015). Prevalência e fatores associados ao declínio cognitivo em idosos com baixa condição econômica: estudo MONIDI. Jornal Brasileiro de Psiquiatria, 64, 187-192.

Oliveira, Paulo Henrique de; Mattos, Inês Echenique. Prevalência e fatores associados à incapacidade funcional em idosos institucionalizados no Município de Cuiabá, Estado de Mato Grosso, Brasil, 2009-2010. Epidemiologia e Serviços de Saúde, v. 21, n. 3, p. 395-406, 2012.

Organização Mundial da Saúde. (2020). Demência.

Organização das Nações Unidas. (2014). A ONU e as pessoas idosas.

Organização Pan-Americana da Saúde. (2019). Demência: número de pessoas afetadas triplicará nos próximos 30 anos. Retirado de https://www.paho.org/bra/index.php?option=com_content\&view=article\&id=5560:dem encianumero-de-pessoas-afetadas-triplicara-nosproximos-30-anos\&Itemid=839.

Prado, M., Nazario, S., Silva, V. H. T., Martinho, A. C. D. O., \&Bergamim, J. S. S. P. (2018). Déficit cognitivo em idosos hospitalizados segundo Mini Exame do Estado Mental (MEEM): Revisão narrativa. Journalof Health Sciences, 20(2), 131-134.

Santos, T. R. A., Lima, D. M., Nakatani, A. Y. K., Pereira, L. V., Leal, G. S., \& Amaral, R. G. (2013). Consumo de medicamentos por idosos, Goiânia, Brasil. Revista de Saúde Pública, 47, 94-103.

Simões, C. C. S. (2016). Relações entre as alterações históricas na dinâmica demográfica brasileira e os impactos decorrentes do processo de envelhecimento da população. Rio de Janeiro: IBGE, Coordenação de População e Indicadores Sociais. Retirado de https://biblioteca.ibge.gov.br/index.php/bibliotecacatalogo?view=detalhes\&id=298579.

Valle, E. A., Castro-Costa, É., Firmo, J. O., Uchoa, E., \& Lima-Costa, M. F. (2009). Estudo de base populacional dos fatores associados ao desempenho no Mini Exame do Estado Mental entre idosos: Projeto Bambuí. Cadernos de Saúde Pública, 25, 918-926.

Vance, D. E., Graham, M. A., Fazeli, P. L., Heaton, K., \&Moneyham, L. (2012). An overview of non-pathological geroneuropsychology: implications for nursing practice and research. The Journal of neuroscience nursing: journal of the American Association of Neuroscience Nurses, $44(1), 43$.

Vargas, L. D. S. D., Lara, M. V. S. D., \& Mello-Carpes, P. B. (2014). Influência da diabetes e a prática de exercício físico e atividades cognitivas e recreativas sobre a função cognitiva e emotividade em grupos de terceira idade. Revista Brasileira de Geriatria e Gerontologia, 17, 867-878.

Wichmann, F. M. A., Couto, A. N., Areosa, S. V. C., \&Montañés, M. C. M. (2013). Grupos de convivência como suporte ao idoso na melhoria da saúde. Revista Brasileira de Geriatria e Gerontologia, 16, 821-832.

World Health Organization. (2005). Envelhecimento ativo: uma política de saúde.

World Health Organization. (2012). Dementia: a public health priority. World Health Organization.

Zangirolami-Raimundo, J., Echeimberg, J. D. O., \& Leone, C. (2018). Tópicos de metodologia de pesquisa: Estudos de corte transversal. J Hum Growth Dev, 28(3), 356-60.

Zortea, B., Gautério-Abreu, D. P., Santos, S. S. C., Silva, B. T. D., Ilha, S., \& Cruz, V. D. (2015). Avaliação cognitiva de pessoas idosas em atendimento ambulatorial. 